\begin{tabular}{|c|c|c|c|}
\hline & Association with $\mathrm{CV}$ risk & $\begin{array}{l}\text { Cox Multivariate } \\
\text { Analysis }\end{array}$ & \\
\hline & Clinical variables & $\mathrm{HR}(95 \% \mathrm{Cl})$ & 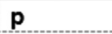 \\
\hline & Age (per $1 \mathrm{SD})$ & $1.752(1.638-1.874)$ & $<2 \times 10-16$ \\
\hline & Sex (male) & $2.399(2.132-2.699)$ & $<2 \times 10-16$ \\
\hline & Diabetes (yes) & $1.378(1.148-1.654)$ & $5.7 \times 10-4$ \\
\hline & BMI (per 1 SD) & $1.149(1.088-1.213)$ & $5.8 \times 10-7$ \\
\hline & SBP (per 1 SD) & $1.121(1.060-1.185)$ & $5.8 \times 10-5$ \\
\hline & ECG variables & & \\
\hline & Heart rate response to recovery (per $1 \mathrm{SD}$ ) & $1.145(1.077-1.217)$ & $1.5 \times 10-5$ \\
\hline & RTc rest (per 1 SD) & $1.099(1.029-1.173)$ & $4.8 \times 10-3$ \\
\hline & RTc exercise (per 1 SD) & $1.068(1.002-1.137)$ & $4.2 \times 10-2$ \\
\hline
\end{tabular}

SD: Standard Deviation, BMI: Body mass index, SBP: Systolic Blood Pressure, HR: Hazzard

cardiovascular (CV) death, suggesting that abnormalities in autonomic balance may precede manifestations of malignant $\mathrm{CV}$ events. Cardiac repolarisation is a critical component in modulating the risk of $\mathrm{CV}$ death. We therefore hypothesised that assessment of autonomic effects on cardiac repolarisation during exercise may help to improve CV risk prediction in the general population as it is more specific for cardiac ventricular pathophysiology compared to the autonomic effects on the sinus node.

Methods A total of 54,203 healthy individuals aged 40-69 years old without prior CV disease who had an exercise stress test from the UK Biobank study were included. HR corrected repolarisation time intervals RTc (an approximation of the QT interval) were measured from the ECG recording at rest (preexercise; RTc_rest), peak-exercise (RTc_ex), and 50s post-exercise recovery (RTc_rec) using a custom build algorithm. We also computed the difference between RTc_ex and RTc_rec $(\mathrm{dRTc})$ as a marker of exercise associated repolarization. We finally performed a follow-up analysis to evaluate the prognostic value of these biomarkers. The endpoints studied were CV death or CV hospitalisation. Associations were tested with the Mann-Whitney and multivariate cox analysis. We then evaluated using multivariate Cox analysis whether individuals in the top $20 \%$ for were significantly more likely to suffer a CV event than those in the bottom $20 \%$.

Results During a median follow-up time of 56 months, 1,460 (2.7\%) individuals reached the endpoint. RTc_ex, RTc_rec, and dRTc were all significantly associated with the endpoint $(\mathrm{p}=4.1 \mathrm{E}-9, \mathrm{p}<2.2 \mathrm{E}-16$, and $\mathrm{p}=5.6 \mathrm{E}-5$, respectively). RTc_ex, remained significantly associated with the endpoint after adjusting for age, diabetes, systolic blood pressure, body mass index, heart rate changes during exercise and recovery, and RTc_rest in the cox proportional hazards model (table 1) with a HR of 1.07 (confidence interval: 1.002-1.137. p=0.04).

Conclusion Assessment of repolarisation time during recovery improves prediction of cardiovascular death and hospitalisation in the general population independently from heart-rate changes. This work supports inclusion of clinical variables to improve personalised diagnostics and demonstrates the importance of evaluating repolarisation during exercise stress testing. Conflict of Interest None

\section{PREDICTING LEFT VENTRICULAR DYSFUNCTION IN A COMMUNITY-BASED COHORT PRESENTING TO HOSPITAL USING CLINICAL CHARACTERISTICS, ECG PARAMETERS AND BIOMARKERS}

${ }^{1}$ Frantisek Nehaj ${ }^{*},{ }^{2}$ Winnie Chua, ${ }^{2}$ Yanish Purmah, ${ }^{3}$ Andre Ziegler, ${ }^{4}$ Russell Davis, ${ }^{4}$ Chetan Varma, ${ }^{4}$ Derek Connolly, ${ }^{2}$ Larissa Fabritz, ${ }^{2}$ Paulus Kirchhof. ${ }^{1}$ Birmingham University; ${ }^{2}$ University of Birmingham; ${ }^{3}$ Roche Diagnostics International AG; ${ }^{4}$ Sandwell and West Birmingham Hospitals

\subsection{6/heartjnl-2019-BCS.22}

Background/Introduction Identifying patients with left ventricular dysfunction (LVDys) remains challenging outside of specialist settings. Abnormalities on the electrocardiogram (ECG) and elevated natriuretic peptides (BNP, NT-proBNP) have been associated with a diagnosis of LVDys or heart failure. As these characteristics will be affected by atrial fibrillation (AF), understanding the behaviour of these predictors in LVDys patients (a)

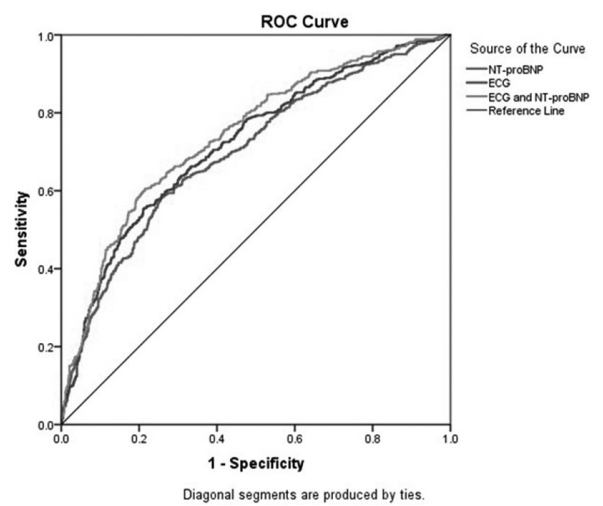

(b)

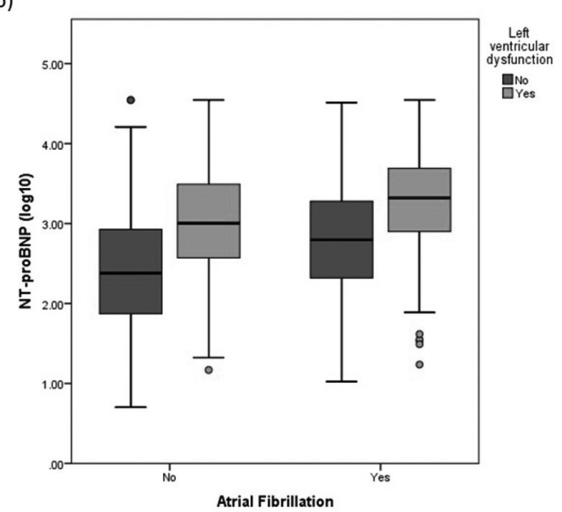


with and without concomitant AF could improve the detection of LVDys.

Purpose To compare the predictive value of clinical characteristics, ECG parameters, and NT-proBNP for prediction of LVDys in patients with and without concomitant AF. We also compared the performance of 6 other common cardiac biomarkers which have been associated with AF.

Methods ECGs from 1487 patients (36\% with LVDys, 39\% female, 44\% with $\mathrm{AF}$, mean [SD] age 68[12] years) presenting to a large teaching hospital were analysed for abnormalities. All patients underwent a standardised transthoracic echocardiogram to determine left ventricular function at baseline. All biomarkers were centrally quantified from plasma using Elisa assays. Using forward selection and logistic regression, 6 ECG parameters (resting heart rate, QRS, QT, QTc durations, presence of AF, and blocks) were modelled and adjusted for clinical characteristics (age, sex, age:sex interaction, history of myocardial infarction, and hypertension). The inclusion of biomarkers was evaluated by examining the C-statistic and 95\% confidence interval.

Results In the overall cohort, after adjustment for clinical characteristics, LVDys was associated with increased heart rate $(\mathrm{OR}$ per 10 beats increase $=1.20$ [95\%CI $=1.12-1.28]$, $\mathrm{p}<0.001)$, prolonged QRS interval $(\mathrm{OR}$ per $10 \mathrm{~ms}$ increase = $1.15[1.10-1.22], \mathrm{p}<0.001)$ and prolonged QTc (OR per 10 $\mathrm{ms}$ increase $=1.08[1.04-1.11], \mathrm{p}<0.001)$. QRS and QTc remained strongly associated with LVDys in patients with and without AF, while heart rate only distinguished between patients with and without LVDys in patients with AF (OR per 10 beats increase $=1.30$ [95\%CI $=1.18-1.42], \mathrm{p}<0.001$ ), but not in patients in sinus rhythm $(p=0.238)$. NT-proBNP levels were significantly elevated in patients with LVDys compared to no LVDys, and further increased in patients with AF (figure 1a). NT-proBNP, (adjusted for clinical characteristics) performed comparably to ECG and clinical characteristics only (C-statistic $=0.70$ [95\%CI $0.67-0.73]$; figure $1 \mathrm{~b})$ to predict LVDys. NT-proBNP outperformed IL-6, Troponin T, GDF15, Creatinine, CRP, and D-dimer even though all biomarkers were elevated in LVDys patients. NT-proBNP combined with ECG parameters improved the C-statistic for patients without AF from $0.70(95 \% \mathrm{CI} 0.66-0.74)$ to $0.73(0.69-0.77)$ and with AF from $0.76(0.73-0.80)$ to 0.77 (0.73-0.81).

Conclusion (s): Two ECG parameters obtained from automated analysis (QRS duration and QTc interval), adjusted for a combination of simple clinical characteristics, robustly identify reduced left ventricular function in unselected patients seen in hospital. NT-proBNP contributes a little to improve the identification of LVDys patients on top of these ECG parameters, with a clear need to adjust normal range in the patients presenting with AF.

Conflict of Interest None

\section{CARDIAC ARREST PATIENTS PRESENTING TO HOSPITALS AT WEEKENDS ARE NOT SUBJECT TO THE WEEKEND EFFECT: INSIGHTS FROM THE ACALM BIG DATA, UNITED KINGDOM}

${ }^{1}$ Rahul Potluri*, ${ }^{2}$ Ranjit More, ${ }^{1}$ Shajil Chalil, ${ }^{2}$ Billal Patel, ${ }^{1}$ Mark Ainslie, ${ }^{3}$ Suresh Chandran. ${ }^{1}$ Blackpool Teaching Hospitals; ${ }^{2}$ Blackpool Teaching Hospital NHS Foundation Trust; ${ }^{3}$ Pennine Acute Hospital NHS Trust

10.1136/heartjnl-2019-BCS.23
Introduction Patients presenting to hospital with a cardiac arrest are associated with significant mortality in the United Kingdom. There is widely debated evidence to support a "weekend-effect" with mortality rates elevated for patients admitted to hospital on the weekend. However, cardiac arrest teams are on call in every acute hospital 24 hours a day, all year round and the evidence for the weekend affect involving cardiac arrests is unclear.

Purpose To investigate the impact of weekend admission on patients presenting with a cardiac arrest to hospital on mortality utilising ACALM big data.

Methods Anonymous information on patients presenting to hospital with a cardiac arrest was obtained from several hospitals in England, UK between 1st January 2000 and 30th June 2014. This data was processed and analysed according to the ACALM (Algorithm for Comorbidities, Associations, Length of stay and Mortality) study protocol. ICD-10 and OPCS-4 codes were used to trace patients coded for cardiac arrest, day of admission, patient demographics, prevalence of co-morbidities and mortality data. Kaplan-Meier survival curves and cox regression analyses adjusted for age, gender, ethnicity, Charlson comorbidity index and the top ten contributors to mortality in the UK (Ischaemic Heart Disease, Heart Failure, Bowel cancer, Breast cancer, Lung cancer, Pneumonia, Cerebrovascular disease, Chronic Obstructive Pulmonary Disease and Dementia).

Results There were 4803 patients who presented with cardiac arrest with the majority, $81 \%$ of patients were admitted on weekdays. Demographics, co-morbidities and mortality for patients admitted on weekdays and weekends are shown in table 1. Figure 1 shows the Kaplan-Meier survival curve for patients discharged according to weekday/weekend discharge. Cox regression model including age, gender, ethnic group, Charlson comorbidity index and the top ten causes of death in the UK showed that weekend presentation did not contribute to increased mortality (OR 1.01;95\%C.I 0.94-1.11; $\mathrm{p}=0.692$ )

Conclusion We have demonstrated that the weekend effect is not shown in patients presenting to hospital with cardiac arrests. Our findings can perhaps be explained by the presence of 24/7 cardiac arrest teams present in acute hospitals.

Conflict of Interest Nil

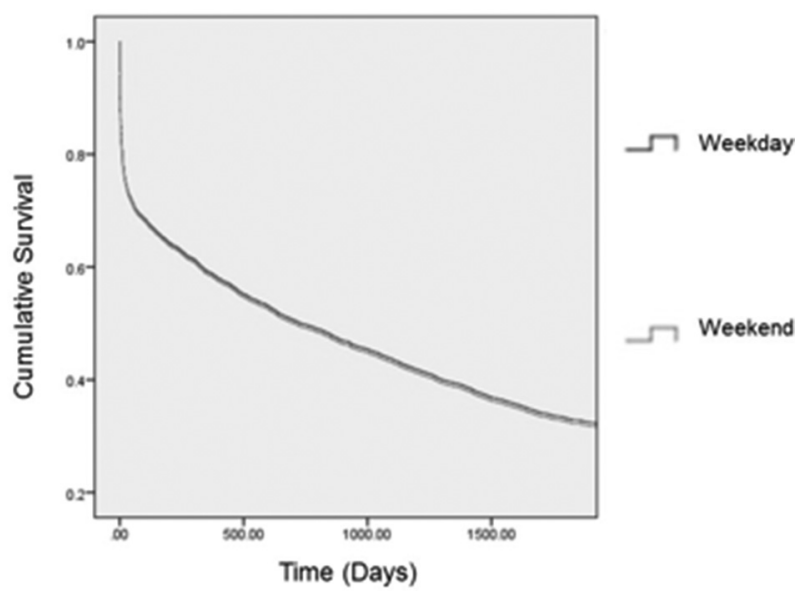

Abstract 24 Figure 15 year survival for cardiac arrest patients presenting to hospital at weekdays vs weekends 